Алгебра и анализ

Том. 15 (2003), вып. 6
St. Petersburg Math. J.

Vol. 15 (2004), No. 6, Pages 837-846 S 1061-0022(04)00834-9

Article electronically published on November 16, 2004

\title{
LOCAL CLASS FIELD THEORY
}

\author{
YU. L. ERSHOV
}

\begin{abstract}
New sufficient conditions for the validity of local class field theory for Henselian valued fields are established. An example is presented to show that these conditions are less restrictive than the applicability of the Neukirch abstract class field theory.
\end{abstract}

\section{$\S 0$. INTRODUCTION}

Local class field theory is treated in many papers, monographs, and textbooks (see, e.g., the bibliography in 11). In the present paper, we suggest yet another exposition based on abstract class field theory 2. (In the author's paper 3, the reader will find a modified presentation of abstract class field theory on the basis of a combination of the approaches by Neukirch and Hasewinkel.)

We recall some definitions and notation. Let $F$ be a field; a subring $R$ of $F$ is called a valuation ring of $F$ if for every element $a \in F^{\times} \rightleftharpoons F \backslash\{0\}$ we have $a \in R$ or $a^{-1} \in R$. Every valuation ring $R$ is local, i.e., has a unique maximal ideal, which will be denoted by $\mathfrak{m}(R)$; the field $F_{R} \rightleftharpoons R / \mathfrak{m}(R)$ is called the residue field of $R$. We denote by $U_{R}$ the multiplicative group of units (invertible elements) of $R$, and by $\Gamma_{R}$ the factor group $F^{\times} / U_{R}$; the group $\Gamma_{R}$ admits a natural linear order, which is determined by the cone $R^{\times} / U_{R} \subseteq \Gamma_{R}$. The mapping $v_{R}: a \mapsto a U_{R}, a \in F^{\times}$, from $F^{\times}$to $\Gamma_{R}$ is called the valuation of $F$ determined by the valuation $\operatorname{ring} R$. Let $U_{R}^{1}$ be the subgroup of $U_{R}$ formed by all elements $a$ in $U_{R}$ such that $a-1 \in \mathfrak{m}(R)$.

The pair $\mathbb{F}=\langle F, R\rangle$, where $F$ is a field and $R$ is a valuation ring of $F$, is called a valued field. Let $\mathbb{F}=\langle F, R\rangle$ and $\mathbb{F}_{0}=\left\langle F_{0}, R_{0}\right\rangle$ be valued fields; if $F$ is a subfield of $F_{0}$ $\left(F \leq F_{0}\right)$ and $R=R_{0} \cap F$, then $\mathbb{F}_{0}$ is an extension of $\mathbb{F}\left(\mathbb{F} \leq \mathbb{F}_{0}\right)$.

If $\mathbb{F}=\langle F, R\rangle \leq \mathbb{F}_{0}=\left\langle F_{0}, R_{0}\right\rangle$, then there exist natural embeddings $F_{R} \rightarrow F_{R_{0}}$ and $\Gamma_{R} \rightarrow \Gamma_{R_{0}}$ of residue fields and valuation groups, respectively. After natural identifications, this allows us to regard $F_{R}$ as a subfield of $F_{R_{0}}\left(F_{R} \leq F_{R_{0}}\right)$ and $\Gamma_{R}$ as a subgroup of $\Gamma_{R_{0}}$; then $v_{R}=v_{R_{0}} \uparrow F^{\times}$. Let $F_{0} / F$ be a finite (algebraic) field extension of degree $n=\left[F_{0}: F\right]$. Then $F_{R_{0}} / F_{R}$ is a finite field extension, and $f \rightleftharpoons\left[F_{R_{0}}: F_{R}\right]$ is called the relative degree of the extension $\mathbb{F} \leq \mathbb{F}_{0}$ of valued fields. The group $\Gamma_{R}$ has a finite index in $\Gamma_{R_{0}}$, and $e \rightleftharpoons\left[\Gamma_{R_{0}}: \Gamma_{R}\right]$ is called the ramification index. We always have the basic inequality $e \cdot f \leq n$. If $e \cdot f=n$ for every finite separable field extension $\mathbb{F}_{0} \geq \mathbb{F}$, then the valued field $\mathbb{F}$ is said to be algebraically complete. A valued field $\mathbb{F}=\langle F, R\rangle$ is Henselian if, for every finite separable extension $F_{0} \geq F$, there is a unique valuation ring $R_{0}$ of $F_{0}$ such that $\mathbb{F} \leq\left\langle F_{0}, R_{0}\right\rangle$. Every algebraically complete valuation is Henselian.

Let $\mathbb{F}=\langle F, R\rangle$ be a valued field such that the residue field $F_{R}$ is perfect and the absolute Galois group $G\left(F_{R}\right)$ of the residue field is isomorphic to $\widehat{\mathbb{Z}}$. Then there is a natural epimorphism $d: G(F) \rightarrow G\left(F_{R}\right)=\widehat{\mathbb{Z}}$ (see [4, Proposition 1.3.3]). If the valuation group

2000 Mathematics Subject Classification. Primary 11R37.

Key words and phrases. Class field theory, Henselian valued field, z-group. 
$\Gamma_{R}$ is a $z$-group (see the definition in $\S 1$ ), then there is a mapping $\widehat{v}: F^{\times} \rightarrow Z_{\Gamma_{R}} \leq \widehat{\mathbb{Z}}$. We say that local class field theory is valid for $\mathbb{F}$ if $\hat{v}: F^{\times} \rightarrow \widehat{\mathbb{Z}}$ is a "Henselian" valuation (see the concluding remarks in [3]) and the $G(F)$-module $F_{\text {sep }}^{\times}\left(F_{\text {sep }}\right.$ is a separable closure of $F$ ) and the mappings $d: G(F) \rightarrow \widehat{\mathbb{Z}}$ and $\hat{v}: F^{\times} \rightarrow \widehat{\mathbb{Z}}$ satisfy the axiom of class field theory (Axiom (6.1) in [2]; Proposition 4.1 below contains the statement of that axiom). If local class field theory is valid for $\mathbb{F}$, then, for every finite Galois extension $F_{0} \geq F$, we have the reciprocity homomorphism $r_{F_{0} / F}: G\left(F_{0} / F\right) \rightarrow H^{0}\left(G\left(F_{0} / F\right), F_{0}^{\times}\right)=F^{\times} / N_{F_{0} / F}\left(F_{0}^{\times}\right)$, which induces an isomorphism $G\left(F_{0} / F\right)^{\mathrm{ab}} \simeq F^{\times} / N_{F_{0} / F}\left(F_{0}^{\times}\right)$.

The following theorem is the main result of the present paper.

Theorem. Suppose that a valued field $\mathbb{F}=\langle F, R\rangle$ satisfies the following four conditions:

1) $\mathbb{F}$ is algebraically complete;

2) the residue field $F_{R}$ is perfect and $G\left(F_{R}\right) \simeq \widehat{\mathbb{Z}}$;

3) the valuation group $\Gamma_{R}$ is a z-group;

4) the maximal unramified extension $\bar{F}$ of $\mathbb{F}$ has dimension at most $1(\operatorname{dim} \bar{F} \leq 1)$; see [5].

Then local class field theory is valid for $\mathbb{F}$.

The proof of this theorem is completed at the end of $\S 4$.

\section{$\S 1 . z$-GROUPS}

Proposition 1.1. For a torsion-free Abelian group $\Gamma$, the following conditions are equivalent:

1) for all $n>0$, the group $\Gamma / n \Gamma$ is cyclic;

2) for every finite torsion-free Abelian extension $\Gamma_{0} \geq \Gamma$, the group $\Gamma_{0} / \Gamma$ is cyclic and $\Gamma=\left[\Gamma_{0}: \Gamma\right] \Gamma_{0}$

3) if $\Pi(\Gamma)$ is the completion of $\Gamma$, then the group $\Pi(\Gamma) / \Gamma$ is locally cyclic.

Proof. 1) $\Longrightarrow 2$ ). Let $n \rightleftharpoons\left[\Gamma_{0}: \Gamma\right]$. Then $n \Gamma \leq n \Gamma_{0} \leq \Gamma$, and $\Gamma_{0} / \Gamma \simeq n \Gamma_{0} / n \Gamma \leq \Gamma / n \Gamma$ is cyclic. Moreover, since $n=\left[\Gamma_{0}: \Gamma\right]=\left[n \Gamma_{0}: n \Gamma\right] \leq[\Gamma: n \Gamma] \leq n$, we have $n \Gamma_{0}=\Gamma$.

2) $\Longrightarrow 3$ ). Let $\gamma_{0}, \ldots, \gamma_{k} \in \Pi(\Gamma)$. Then $\Gamma_{0} \rightleftharpoons\left\langle\gamma_{0}, \ldots, \gamma_{k}, \Gamma\right\rangle \geq \Gamma$ is a finite torsionfree Abelian extension, and $\Gamma_{0} / \Gamma=\left\langle\gamma_{0}+\Gamma, \ldots, \gamma_{k}+\Gamma\right\rangle$ is cyclic.

$3) \Longrightarrow 1)$. Since $\Gamma$ is torsion-free, we have $\Gamma \simeq n \Gamma$, whence $\Pi(\Gamma)=\Pi(n \Gamma)$ and $\Pi(n \Gamma) \geq n \Gamma$. Consequently, $\Gamma / n \Gamma \leq \Pi(n \Gamma) / n \Gamma$ is a locally cyclic group of a bounded period at most $n$. Thus, $\Gamma / n \Gamma$ is cyclic (and $[\Gamma: n \Gamma] \mid n$ ).

We say that a torsion-free Abelian group $\Gamma$ is a $z$-group if $[\Gamma: n \Gamma]=n$ for every positive integer $n$.

Proposition 1.2. For a torsion-free Abelian group $\Gamma$, the following two conditions are equivalent:

1) $\Gamma$ is a z-group;

2) for every $n>0$, the group $\Gamma / n \Gamma$ is a cyclic group of order $n$.

Proof. 1) $\Longrightarrow 2$ ). Assume the contrary. Then there is a positive integer $n$ such that the group $\Gamma / n \Gamma$ is not cyclic (but $|\Gamma / n \Gamma|=n$ ). Consequently, there exists a prime $p$ such that the group $\Gamma / n \Gamma$ has a factor group isomorphic to $C_{p} \times C_{p}$ (here $C_{p}$ is a cyclic group of order $p)$. Let $\Gamma_{0}(\geq n \Gamma)$ be a subgroup of $\Gamma$ such that $\Gamma / \Gamma_{0} \simeq C_{p} \times C_{p}$. It follows that $\Gamma_{0} \geq p \Gamma$ and $p^{2}=\left|\Gamma / \Gamma_{0}\right| \leq|\Gamma / p \Gamma|=p$, a contradiction.

$2) \Longrightarrow 1$ ). This is obvious.

Let $\Gamma$ be a $z$-group. Then there is a natural homomorphism $\pi_{\Gamma}: \Gamma \rightarrow \varliminf_{n>0} \Gamma / n \Gamma \simeq$ $\widehat{\mathbb{Z}}$. We put $Z_{\Gamma} \rightleftharpoons \pi(\Gamma) \leq \widehat{\mathbb{Z}}$. 
Proposition 1.3. a) $\operatorname{Ker} \pi_{\Gamma}$ is the maximal divisible subgroup of $\Gamma$;

b) $Z_{\Gamma}$ is a pure subgroup of $\widehat{\mathbb{Z}}$ and $\widehat{\mathbb{Z}} / Z_{\Gamma}$ is divisible.

Proof. Since Ker $\pi_{\Gamma}=\bigcap_{n>0} n \Gamma$, statement a) follows immediately from the fact that $\Gamma$ is an Abelian torsion-free group (and, therefore, is uniquely divisible).

Let $m>0$. Then the natural projection $p_{m}: \widehat{\mathbb{Z}}=\lim _{n>0} \Gamma / n \Gamma \rightarrow \Gamma / m \Gamma \simeq C_{m}$ is an epimorphism, so that $\operatorname{Ker} p_{m}=m \widehat{\mathbb{Z}}$. It follows that ${ }_{\widehat{m} \widehat{\mathbb{Z}}}^{n} \cap Z_{\Gamma}=m Z_{\Gamma}$ and, therefore, $Z_{\Gamma}$ is a pure subgroup of $\widehat{\mathbb{Z}}$. If $a \in \widehat{\mathbb{Z}}$ and $m>0$, then $a+m \widehat{\mathbb{Z}}=b+m \widehat{\mathbb{Z}}$ for an appropriate $b \in Z_{\Gamma}$ (because $\widehat{\mathbb{Z}} / m \widehat{\mathbb{Z}}=Z_{\Gamma} / m \widehat{\mathbb{Z}} \cap Z_{\Gamma}$ ). However, in this case there exists $c \in \widehat{\mathbb{Z}}$ such that $a-b=m c$. Consequently, the group $\widehat{\mathbb{Z}} / Z_{\Gamma}$ is divisible.

Let $\mathbb{F}=\langle F, R\rangle$ be a valued field such that the valuation group $\Gamma_{R}$ is a $z$-group. Then we have a homomorphism $\hat{v} \rightleftharpoons \pi_{\Gamma_{R}} v_{R}: F^{\times} \rightarrow Z_{\Gamma_{R}} \leq \widehat{\mathbb{Z}}$.

Proposition 1.4. Let $\mathbb{F}=\langle F, R\rangle$ be a valued field satisfying conditions 1) and 3) of the theorem. Then the following relations are valid for every finite extension $\mathbb{F}_{0}=\left\langle F_{0}, R_{0}\right\rangle \geq$ $\mathbb{F}$ :

1) $v_{R}\left(N_{F_{0} / F}\left(F_{0}^{\times}\right)\right)=f \Gamma_{R}$

2) $\hat{v}\left(N_{F_{0} / F}\left(F_{0}^{\times}\right)\right)=f Z_{\Gamma_{R}}$, where $f \rightleftharpoons\left[F_{R_{0}}: F_{R}\right]$ is the relative degree of the extension $\mathbb{F}_{0} \geq \mathbb{F}$.

Proof. If $n \rightleftharpoons\left[F_{0}: F\right]$ and $e \rightleftharpoons\left[\Gamma_{R_{0}}: \Gamma_{R}\right]$ is the ramification index of the extension $\mathbb{F}_{0} \geq \mathbb{F}$, then the algebraic completeness of $\mathbb{F}$ (condition 1)) implies the relation $n=e \cdot f$. Since $\Gamma_{R}$ is a $z$-group, from the relation $\left[\Gamma_{R_{0}}: \Gamma_{R}\right]=e$ it follows that $\Gamma_{R}=e \Gamma_{R_{0}}$ (see Proposition 1.2).

For each $a_{0} \in F_{0}^{\times}$we have $v_{R_{0}}\left(N_{F_{0} / F}\left(a_{0}\right)\right)=n v_{R_{0}}\left(a_{0}\right)$, whence $v_{R}\left(N_{F_{0} / F}\left(F_{0}^{\times}\right)\right)$ $=v_{R_{0}}\left(N_{F_{0} / F}\left(F_{0}^{\times}\right)\right)=n \Gamma_{R_{0}} \leq \Gamma_{R}$. However, $n \Gamma_{R_{0}}=(f \cdot e) \Gamma_{R_{0}}=f\left(e \Gamma_{R_{0}}\right)=f \Gamma_{R}$. Statement 1) is verified. Statement 2) follows directly from 1).

Corollary. If a valued field $\mathbb{F}$ satisfies conditions 1), 2), and 3) of the theorem, then the homomorphism

$$
\hat{v}: F^{\times} \rightarrow Z_{\Gamma_{R}} \leq \widehat{\mathbb{Z}}
$$

is a "Henselian" valuation in the sense of abstract class field theory $[3]$.

\section{§2. UNRAMIFIED EXTENSIONS}

Proposition 2.1. Let $\mathbb{F}=\langle F, R\rangle \leq \mathbb{F}^{\prime}=\left\langle F^{\prime}, R^{\prime}\right\rangle$ be a finite extension of Henselian valued fields such that $F_{R^{\prime}}$ is a separable extension of $F_{R}$ and $\left[F^{\prime}: F\right]=\left[F_{R^{\prime}}: F_{R}\right]$. Then $N_{F^{\prime} / F}\left(U_{R^{\prime}}^{1}\right)=U_{R}^{1}$. If $N_{F_{R^{\prime}} / F_{R}}\left(F_{R^{\prime}}^{\times}\right)=F_{R}^{\times}$, then $N_{F^{\prime} / F}\left(U_{R^{\prime}}\right)=U_{R}$.

Proof. Suppose $\alpha \in F_{R^{\prime}}^{\times}$and $F_{R^{\prime}}=F_{R}(\alpha)$. Let $f \in F_{R}[x]$ be a minimal polynomial of $\alpha$ over $F_{R}$, and let $g=x^{n}+b_{1} x^{n-1}+\cdots+b_{n} \in R[x]$ be a unitary polynomial such that its reduction $\bar{g} \in F_{R}[x]$ coincides with $f$. By the Hensel lemma, there exists $a \in R^{\prime}$ such that $g(a)=0$ and $\bar{a}\left(\rightleftharpoons a+\mathfrak{m}\left(R^{\prime}\right)\right)=\alpha$. Then $N_{F^{\prime} / F}(a)=(-1)^{n} b_{n}$ (because $g$ is irreducible over $F$ and $F^{\prime}=F(a)$ ). Let $u \in U_{R}^{1}$ (i.e., $u \in R$ and $\bar{u}=1$ ). Then $\bar{g}_{1}=f$, where $g_{1} \rightleftharpoons x^{n}+b_{1} x^{n-1}+\cdots+b_{n-1} x+u b_{n}$, and, again by the Hensel lemma, there exists an element $a_{1} \in R^{\prime}$ such that $\bar{a}_{1}=\alpha$ and $g_{1}\left(a_{1}\right)=0$. As above, we have $N_{F^{\prime} / F}\left(a_{1}\right)=(-1)^{n} u b_{n}$; consequently, $N_{F^{\prime} / F}\left(a_{1} a^{-1}\right)=N_{F^{\prime} / F}\left(a_{1}\right) N_{F^{\prime} / F}(a)^{-1}=u$. Since $\overline{a_{1} a^{-1}}=\alpha \alpha^{-1}=1$, we have $a_{1} a^{-1} \in U_{R^{\prime}}^{1}$ and $u=N_{F^{\prime} / F}\left(a_{1} a^{-1}\right) \in N_{F^{\prime} / F}\left(U_{R^{\prime}}^{1}\right)$.

If, moreover, $N_{F_{R}^{\prime} / F_{R}}\left(F_{R^{\prime}}^{\times}\right)=F_{R}^{\times}$, then for every $u \in U_{R}$ there exists $v \in U_{R^{\prime}}$ such that $u^{-1} N_{F^{\prime} / F}(v) \in U_{R}^{1}$. Indeed, $\bar{u} \in F_{R}^{\times}$. Let $\alpha \in F_{R^{\prime}}^{\times}$be such that $N_{F_{R^{\prime}} / F_{R}}(\alpha)=\bar{u}$, and let $v \in U_{R^{\prime}}$ be such that $\bar{v}=\alpha$. Then $\overline{N_{F^{\prime}} / F}(v)=N_{F_{R^{\prime}} / F_{R}}(\bar{v})=N_{F_{R^{\prime}} / F_{R}}(\alpha)=\bar{u}$. 
Thus, $u^{-1} N_{F^{\prime} / F}(v) \in U_{R}^{1}$. As has been proved above, there is $a \in U_{R^{\prime}}^{1}$ such that $N_{F^{\prime} / F}(a)=u^{-1} N_{F^{\prime} / F}(v)$, and then $u=N_{F^{\prime} / F}\left(v a^{-1}\right) \in N_{F^{\prime} / F}\left(U_{R}\right)$.

Corollary. If a valued field $\mathbb{F}$ satisfies conditions 1) and 2) of the theorem, then for every finite unramified extension $\mathbb{F} \leq \mathbb{F}^{\prime}$ we have $N_{F^{\prime} / F}\left(U_{R^{\prime}}\right)=U_{R}$.

Indeed, since $\mathbb{F}$ is algebraically complete and $\mathbb{F}^{\prime} \geq \mathbb{F}$ is unramified, $\left[F^{\prime}: F\right]=$ $\left[F_{R^{\prime}}: F_{R}\right]$. Since $F_{R}$ is perfect, the conditions of the proposition are fulfilled. Furthermore, using isomorphism $G\left(F_{R}\right) \simeq \widehat{\mathbb{Z}}$ and the fact that $F_{R}$ is perfect, we conclude that $\operatorname{dim} F_{R} \leq 1$ (see [5 Chapter II, §3, Proposition 6(b)]); in particular, $N_{F_{R^{\prime}} / F_{R}}\left(F_{R^{\prime}}^{\times}\right)=F_{R}^{\times}$.

We now verify the class field theory axiom for unramified extensions.

Proposition 2.2. Let $\mathbb{F}$ be a valued field satisfying conditions 1)-3) of the theorem. Then for every unramified cyclic extension $\mathbb{F}_{0} \geq \mathbb{F}$ we have $H^{0}\left(G, F_{0}^{\times}\right) \simeq G$ and $H^{-1}\left(G, F_{0}^{\times}\right)=$ 1 , where $G \rightleftharpoons G\left(F_{0} / F\right)$.

Proof. The group $H^{-1}\left(G, F_{0}^{\times}\right)$is trivial by virtue of Hilbert's Theorem 90 [2].

Consider the exact sequence

$$
1 \longrightarrow U_{R} \longrightarrow F^{\times} \stackrel{v_{R}}{\longrightarrow} \Gamma_{R} \longrightarrow 1,
$$

which yields the exact sequence

$$
\begin{array}{cc}
1 \longrightarrow U_{R} / N_{F_{0} / F}\left(F_{0}^{\times}\right) \cap U_{R} \longrightarrow F^{\times} / N_{F_{0} / F}\left(F_{0}^{\times}\right) \\
\longrightarrow \Gamma_{R} / v_{R}\left(N_{F_{0} / F}\left(F_{0}^{\times}\right)\right) \longrightarrow c & 1 .
\end{array}
$$

Since $N_{F_{0} / F}\left(F_{0}^{\times}\right) \cap U_{R}=N_{F_{0} / F}\left(U_{R_{0}}\right)$, we have $U_{R}=N_{F_{0} / F}\left(U_{\left.R_{0}\right)}\right.$ by Proposition 2.1 . Consequently, we arrive at an isomorphism

$$
F^{\times} / N_{F_{0} / F}\left(F_{0}^{\times}\right) \simeq \Gamma_{R} / v_{R}\left(N_{F_{0} / F}\left(F_{0}^{\times}\right)\right) .
$$

Let $n \rightleftharpoons|G|\left(=\left[F_{0}: F\right]=\left[F_{R_{0}}: F_{R}\right]\right)$. Since $F_{0} \geq F$ is an unramified extension, the relative degree $f$ of the extension $F_{0} \geq F$ is equal to $n$. By Proposition 1.4, 1), we have $v_{R}\left(N_{F_{0} / F}\left(F_{0}^{\times}\right)\right)=n \Gamma_{R}$, and since $\Gamma$ is a $z$-group, the group $\Gamma / n \Gamma \simeq C_{n}$ is cyclic of order $n$. Thus,

$$
H^{0}\left(G, F_{0}^{\times}\right)=F^{\times} / N_{F_{0} / F}\left(F_{0}^{\times}\right) \simeq \Gamma / n \Gamma \simeq C_{n} \simeq G .
$$

Remark. Under the conditions of Proposition 2.2, the axiom $U$ (i.e., $H^{0}\left(G, U_{R_{0}}\right)=1$ and $H^{-1}\left(G, U_{R_{0}}\right)=1$; see [3] $)$ is fulfilled.

Indeed, consider the exact sequence of $G$-modules

$$
1 \longrightarrow U_{R_{0}} \longrightarrow F_{0}^{\times} \stackrel{u_{R_{0}}}{\longrightarrow} \Gamma_{R_{0}} \longrightarrow 1
$$

and the corresponding exact sequence of cohomology groups

$$
\begin{aligned}
H^{0}\left(G, U_{R_{0}}\right) & \longrightarrow H^{0}\left(G, F_{0}^{\times}\right) \longrightarrow H^{0}\left(G, \Gamma_{R_{0}}\right) \\
& \longrightarrow H^{1}\left(G, U_{R_{0}}\right) \longrightarrow H^{1}\left(G, F_{0}^{\times}\right) .
\end{aligned}
$$

By the corollary to Proposition 2.1, we have $H^{0}\left(G, U_{R_{0}}\right)=1$, and, by Hilbert's Theorem $90, H^{1}\left(G, F_{0}^{\times}\right)=1$. Therefore, we have the exact sequence

$$
1 \longrightarrow H^{0}\left(G, F_{0}^{\times}\right) \longrightarrow H^{0}\left(G, \Gamma_{R_{0}}\right) \longrightarrow H^{1}\left(G, U_{R_{0}}\right) \longrightarrow 1 .
$$

Proposition 2.2 implies that $H^{0}\left(G, F_{0}^{\times}\right) \simeq G \simeq C_{n}$. Since $G$ acts trivially on $\Gamma_{R_{0}}$, we obtain $N\left(\Gamma_{R_{0}}\right)=n \Gamma_{R_{0}}$ (in additive notation), and $H^{0}\left(G, \Gamma_{R_{0}}\right) \simeq \Gamma_{R_{0}} / n \Gamma_{R_{0}} \simeq \Gamma_{R} / n \Gamma_{R} \simeq$ $C_{n}$. Consequently, $H^{-1}\left(G, U_{R_{0}}\right) \simeq H^{1}\left(G, U_{R_{0}}\right)=1$; the fact that the $H^{0}\left(G, U_{R_{0}}\right)=1$ has already been mentioned. 


\section{§3. Totally RAMified CYCliC EXTEnsions}

Proposition 3.1. Let $\mathbb{F}=\langle F, R\rangle$ be a Henselian valued field such that $\Gamma_{R}$ is a z-group. If $\mathbb{F}_{0}=\left\langle F_{0}, R_{0}\right\rangle \geq \mathbb{F}$ is a cyclic totally ramified extension of $\mathbb{F}$, then $G \simeq H^{-1}\left(G, U_{R_{0}}\right)$, where $G \rightleftharpoons G\left(F_{0} / F\right)$.

Proof. Let $n \rightleftharpoons\left[F_{0}: F\right]=\left[\Gamma_{R_{0}}: \Gamma_{R}\right]$. Then $\Gamma_{R}=n \Gamma_{R_{0}}$ and $\Gamma_{R_{0}} / \Gamma_{R}$ is a cyclic group of order $n$ (i.e., $\Gamma_{R_{0}} / \Gamma_{R} \simeq G=G\left(F_{0} / F\right)$ ). Let $\gamma_{0} \in \Gamma_{R_{0}}$ be an element such that $\gamma_{0}>0$ and $\left\langle\gamma_{0}+\Gamma_{R}\right\rangle=\Gamma_{R_{0}} / \Gamma_{R}$. We put $\gamma \rightleftharpoons n \gamma_{0}, \gamma \in \Gamma_{R}$. Let $\pi_{0} \in R_{0}^{\times}$be an element such that $v_{R_{0}}\left(\pi_{0}\right)=\gamma_{0}$. Then for $\pi \rightleftharpoons N_{F_{0} / F}\left(\pi_{0}\right) \in R^{\times}$we have $v_{R}(\pi)=v_{R_{0}}(\pi)=$ $v_{R_{0}}\left(N_{F_{0} / F}\left(\pi_{0}\right)\right)=n \gamma_{0}=\gamma$.

For $\tau \in G$, we put $\chi(\tau) \rightleftharpoons \pi_{0}^{\tau-1} \cdot V\left(F_{0} / F\right)$, where $V\left(F_{0} / F\right) \rightleftharpoons\left\langle u^{\sigma-1}\right| u \in U_{R_{0}}$, $\sigma \in G\rangle \leq U_{R_{0}}$. We note that $N_{F_{0} / F}\left(\pi_{0}^{\tau-1}\right)=N_{F_{0} / F}\left(\pi_{0}\right)^{\tau-1}=1$. Consequently, $\chi(\tau) \in$ ${ }_{N} U_{R_{0}} / V\left(F_{0} / F\right)$ (the inclusion $V\left(F_{0} / F\right) \leq{ }_{N} U_{R_{0}}$ can also be verified easily). We recall that ${ }_{N} U_{R_{0}}=\left\{u \mid u \in U_{R_{0}}, N_{F_{0} / F}(u)=1\right\}$.

We check that $\chi$ is independent of the choice of $\pi_{0}$. If $v_{R_{0}}\left(\pi_{1}\right)=\gamma_{0}=v_{R_{0}}\left(\pi_{0}\right)$, then $\pi_{1}=\pi_{0} u$ for an appropriate $u \in U_{R_{0}}$, so that we have $\pi_{1}^{\tau-1}=\left(\pi_{0} u\right)^{\tau-1}=\pi_{0}^{\tau-1} u^{\tau-1}$ and $u^{\tau-1} \in V\left(F_{0} / F\right)$.

We prove that $\chi$ is a homomorphism from $G$ to ${ }_{N} U_{R_{0}} / V\left(F_{0} / F\right)$. Let $\tau_{0}, \tau_{1} \in G$. Then $\tau_{0} \tau_{1}-1=\tau_{0} \tau_{1}-\tau_{0}+\tau_{0}-1$ and $\pi_{0}^{\tau_{0} \tau_{1}-1}=\left(\pi_{0}^{\tau_{0}}\right)^{\tau_{1}-1} \pi_{0}^{\tau_{0}-1}$.

However, $v_{R_{0}}\left(\pi_{0}^{\tau_{0}}\right)=v_{R_{0}}\left(\pi_{0}\right)=\gamma_{0}$, whence $\left(\pi_{0}^{\tau_{0}}\right)^{\tau_{1}-1} \equiv \pi_{0}^{\tau_{1}-1} \bmod V\left(F_{0} / F\right)$ and $\pi_{0}^{\tau_{0} \tau_{1}-1} V\left(F_{0} / F\right)=\pi_{0}^{\tau_{0}-1} V\left(F_{0} / F\right) \cdot \pi_{0}^{\tau_{1}-1} V\left(F_{0} / F\right)$. Thus, $\chi\left(\tau_{0} \tau_{1}\right)=\chi\left(\tau_{0}\right) \chi\left(\tau_{1}\right)$.

Now, we prove that $\chi$ is an epimorphism. Let $u \in{ }_{N} U_{R_{0}}$, i.e., $N_{F_{0} / F}(u)=1$. Then, by Hilbert's Theorem 90, we have $u=a_{0}^{\tau_{0}-1}$ for some $\tau_{0} \in G$ such that $\left\langle\tau_{0}\right\rangle=G$ and for an appropriate $a_{0} \in F_{0}^{\times}$. Let $\gamma_{1} \rightleftharpoons v_{R_{0}}\left(a_{0}\right)$. Then we can find $\gamma^{\prime} \in \Gamma_{R}$ and $0 \leq k<n$ such that $\gamma_{1}=\gamma^{\prime}+k \gamma_{0}$ (because $\left.\Gamma_{R_{0}} / \Gamma_{R}=\left\langle\gamma_{0}+\Gamma_{R}\right\rangle\right)$. Let $a \in F^{\times}$be an element such that $v_{R}(a)=\gamma^{\prime}$. Then $v_{R_{0}}\left(a_{0}\left(a \pi_{0}^{k}\right)^{-1}\right)=0$ and $a_{0}=v a=\pi_{0}^{k}$ for $v \rightleftharpoons a_{0}\left(a \pi_{0}^{k}\right)^{-1} \in U_{R_{0}}$. We have $a_{0}^{\tau_{0}-1}=\left(v a \pi_{0}^{k}\right)^{\tau_{0}-1}=v^{\tau_{0}-1}\left(\pi_{0}^{k}\right)^{\tau_{0}-1} \equiv\left(\pi_{0}^{k}\right)^{\tau_{0}-1} \equiv$ $\pi_{0}^{\tau_{0}^{k}-1} \bmod V\left(F_{0} / F\right)$. Consequently, $v V\left(F_{0} / F\right)=a_{0}^{\tau_{0}-1} V\left(F_{0} / F\right)=\chi\left(\tau_{0}^{k}\right) \in \chi(G)$ and $\chi(G)={ }_{N} U_{R_{0}} / V\left(F_{0} / F\right)$.

We prove that $\chi$ is a monomorphism. If $G=\left\langle\tau_{0}\right\rangle$, then $V\left(F_{0} / F\right)=U_{R_{0}}^{\tau_{0}-1}=$ $\left\{u^{\tau_{0}-1} \mid u \in U_{R_{0}}\right\}$. Indeed, $u^{\tau_{0}^{k}-1}=u^{\left(\tau_{0}^{k-1}+\cdots+1\right)\left(\tau_{0}-1\right)}=v^{\tau_{0}-1}$, where $v \rightleftharpoons u^{\tau_{0 k}}$ and $\tau_{0 k} \rightleftharpoons \tau_{0}^{k-1}+\cdots+1$. Let $\pi_{0}^{\tau_{0}^{k}-1} \in V\left(F_{0} / F\right)$, i.e., $\pi_{0}^{\tau_{0}^{k}-1}=u^{\tau_{0}-1}$ for an appropriate $u \in U_{R_{0}}$. Then $\pi_{0}^{\tau_{0}^{k}-1}=\left(\pi_{0}^{\tau_{0 k}}\right)^{\tau_{0}-1}=u^{\tau_{0}-1}$ and $\left(\pi_{0}^{\tau_{0 k}} u^{-1}\right)^{\tau_{0}-1}=1$. Consequently, $\pi_{0}^{\tau_{0 k}} u^{-1} \in F_{0}^{\tau_{0}}=F_{0}^{G}=F$ and $v_{R_{0}}\left(\pi_{0}^{\tau_{0 k}} u^{-1}\right)=v_{R_{0}}\left(\pi_{0}^{\tau_{0 k}}\right)=k v_{R_{0}}\left(\pi_{0}\right)=k \gamma_{0} \in \Gamma_{R}=$ $n \Gamma_{R_{0}}$. This shows that $n$ divides $k$ and $\pi^{r_{0 k}} \tau_{0}^{k}=1$.

Thus, $\chi$ is an isomorphism between $G$ and ${ }_{N} U_{R_{0}} / V\left(F_{0} / F\right)$. But $V\left(F_{0} / F\right)=I_{G} U_{R_{0}}(\rightleftharpoons$ $\left.\left\langle u^{\sigma-1} \mid u \in U_{R_{0}}, \sigma \in G\right\rangle\right)$, and therefore

$$
{ }_{N} U_{R_{0}} / V\left(F_{0} / F\right)=H^{-1}\left(G, U_{R_{0}}\right)\left(\rightleftharpoons{ }_{N} U_{R_{0}} / I_{G} U_{R_{0}}\right) .
$$

Proposition 3.2. Let $\mathbb{F}=\langle F, R\rangle$ be a valued field satisfying conditions 1)-4) of the theorem. If $\mathbb{F}_{0}=\left\langle F_{0}, R_{0}\right\rangle \geq \mathbb{F}$ is a cyclic totally ramified extension, then $G\left(F_{0} / F\right) \simeq$ $H^{0}\left(G, U_{R_{0}}\right)=H^{0}\left(G, F_{0}^{\times}\right)$.

Proof. Let $G \rightleftharpoons G\left(F_{0} / F\right), G=\langle\tau\rangle$, and let $n=|G|=\left[F_{0}: F\right]$. Suppose $\mathbb{F}_{1}=\left\langle F_{1}, R_{1}\right\rangle$ is an unramified extension of $\mathbb{F}$ of degree $m$ divisible by $n, G_{1} \rightleftharpoons G\left(F_{1} / F\right)$, and $G_{1}=\langle\psi\rangle$. Let $F_{2} \rightleftharpoons F_{0} F_{1}=F_{0} \otimes_{F} F_{1}$, and let $R_{2}$ be a valuation ring of $F_{2}$ dominating $R$ (and $R_{0}$ and $\left.R_{1}\right)$. Then $G\left(F_{2} / F\right) \simeq G \times G_{1}=\langle\tau\rangle \times\langle\psi\rangle$, and $\mathbb{F}_{2} \rightleftharpoons\left\langle F_{2}, R_{2}\right\rangle$ is totally ramified over $\mathbb{F}_{1}$ and unramified over $\mathbb{F}_{0}$. We have $G\left(F_{2} / F_{1}\right) \simeq G=\langle\tau\rangle, G\left(F_{2} / F_{0}\right) \simeq G_{1}=\langle\psi\rangle$, $F_{2}^{\psi}=F_{0}$, and $F_{2}^{\tau}=F_{1}$. 
We introduce a group $U_{R}^{F_{2}}\left(\leq U_{R}\right)$ and a homomorphism $\rho_{\psi}: U_{R}^{F_{2}} \rightarrow H^{-1}\left(G, U_{R_{2}}\right)$ as follows:

$U_{R}^{F_{2}} \rightleftharpoons\left\{u \mid u \in U_{R}\right.$ is such that there exists $v \in U_{R_{2}}$ with $\left.N_{F_{2} / F_{1}}(v)=u\right\}$;

if $u \in U_{R}^{F_{2}}$ and $v$ is an element of $U_{R_{2}}$ such that $N_{F_{2} / F_{1}}(v)=u$, then

$$
\rho_{\psi}(u) \rightleftharpoons v^{1-\psi} V\left(F_{2} / F_{1}\right) .
$$

We prove that $\rho_{\psi}$ is well defined. If $v^{\prime} \in U_{R_{2}}$ and $N_{F_{2} / F_{1}}\left(v^{\prime}\right)=u$, then $N_{F_{2} / F_{1}}\left(w^{-1}\right)=$ $u u^{-1}=1$ for $w \rightleftharpoons v^{\prime} v^{-1}$. Consequently, by Hilbert's Theorem 90, $w=w_{0}^{\tau-1}$ for an appropriate $w_{0} \in U_{R_{2}}$. But in this case we have $v^{1-\psi}=(v w)^{1-\psi}=v^{1-\psi} w_{0}^{(\tau-1)(1-\psi)}$ and $w_{0}^{(\tau-1)(1-\psi)}=\left(w_{0}^{(1-\psi)}\right)^{\tau-1} \in V\left(F_{2} / F_{1}\right)$. Therefore, $v^{1-\psi} V\left(F_{2} / F_{1}\right)=v^{1-\psi} V\left(F_{2} / F_{1}\right)$, and we see that $\rho_{\psi}$ is well defined.

Now, we prove that $\rho_{\psi}$ is a homomorphism. If $u_{0}, u_{1} \in U_{R}^{F_{2}}$ and $v_{0}, v_{1} \in U_{R_{2}}$ are such that $N_{F_{2} / F_{1}}\left(v_{i}\right)=u_{i}(i=0,1)$, then $v_{0} v_{1} \in U_{R_{2}}, N_{F_{2} / F_{1}}\left(v_{0} v_{1}\right)=N_{F_{2} / F_{1}}\left(v_{0}\right) N_{F_{2} / F_{1}}\left(v_{1}\right)$ $=u_{0} u_{1}$, and $\left(v_{0} v_{1}\right)^{1-\psi}=v_{0}^{1-\psi} v_{1}^{1-\psi}$. Hence, $\rho_{\psi}\left(u_{0} u_{1}\right)=\rho_{\psi}\left(u_{0}\right) \cdot \rho_{\psi}\left(u_{1}\right)$, i.e., $\rho_{\psi}$ is a homomorphism.

Let $\psi^{\prime} \rightleftharpoons \psi \tau \in G\left(F_{2} / F\right)=G \times G_{1}=\langle\tau\rangle \times\langle\psi\rangle$. Observe that $\left|\psi^{\prime}\right|=|\psi|=m$ because $|\tau|=n$ divides $m$. We put $F_{0}^{\prime} \rightleftharpoons F_{2}^{\psi^{\prime}}$ and $R_{0}^{\prime} \rightleftharpoons R_{2} \cap F_{0}^{\prime}$. It is easy to check that $\mathbb{F}_{2}$ is unramified over $\mathbb{F}_{0}^{\prime}=\left\langle F_{0}^{\prime}, R_{0}^{\prime}\right\rangle$.

We define a homomorphism $\rho_{\psi^{\prime}}: U_{R}^{F_{2}} \rightarrow H^{-1}\left(G, U_{R_{2}}\right)$ in the same way as $\rho_{\psi}$ :

for $u \in U_{R}^{F_{2}}$, we take $v \in U_{R_{2}}$ such that $N_{F_{2} / F_{1}}(v)=u$ and put

$$
\rho_{\psi^{\prime}}(u) \rightleftharpoons v^{1-\psi^{\prime}} V\left(F_{0} / F_{1}\right) .
$$

We note that $\rho_{\psi^{\prime}}=\rho_{\psi}$. Let $v \in U_{R_{2}}$ and $u=N_{F_{2} / F_{1}}(v) \in U_{R}$. Then $v^{1-\psi^{\prime}}=v^{1-\psi \tau}=$ $v^{1-\tau+\tau-\tau \psi}=\left(v^{1-\tau}\right)\left(v^{\tau}\right)^{1-\psi}$. Since $v^{1-\tau} \in V\left(F_{2} / F_{1}\right)$ and $N_{F_{2} / F_{1}}\left(v^{\tau}\right)=N_{F_{2} / F_{1}}(v)^{\tau}=$ $u^{\tau}=u$, we have $\rho_{\psi^{\prime}}(u)=v^{1-\psi^{\prime}} V\left(F_{2} / F_{1}\right)=v^{1-\psi} V\left(F_{2} / F_{1}\right)=\rho_{\psi}(u)$.

Now, we verify that $\rho_{\psi}$ is an epimorphism. By Proposition 3.1, the mapping $\chi: G \rightarrow$ $H^{-1}\left(G_{0}, U_{R_{2}}\right)$ defined by $\chi\left(\tau^{\prime}\right) \rightleftharpoons \pi_{0}^{\tau^{\prime}-1} V\left(F_{2} / F_{1}\right)$ for $\tau^{\prime} \in G_{0}=\langle\tau\rangle$ is an isomorphism; here $\pi_{0} \in R_{0} \leq R_{2}$ is chosen as in the proof of Proposition $3.1\left(v_{R_{0}}\left(\pi_{0}\right)=\gamma_{0}\right)$. It follows that the element $\pi_{0}^{\tau-1} V\left(F_{2} / F_{1}\right)$ is a generator of the cyclic group $H^{-1}\left(G, U_{\left.R_{2}\right)}(\simeq G)\right.$.

Let $\pi_{0}^{\prime} \in R_{0}^{\prime}$ be such that $v_{R_{0}^{\prime}}\left(\pi_{0}^{\prime}\right)=\gamma_{0}$. Then for $v \rightleftharpoons \pi_{0}^{\prime} \pi_{0}^{-1}$ we have $v_{R_{2}}(v)=1$, $v \in U_{R_{2}}$, and $\pi_{0}^{\prime}=\pi_{0} v$. Since $\pi_{0}^{\prime} \in F_{0}^{\prime}$, we obtain

$$
\pi_{0}^{\prime}=\pi_{0}^{\prime \psi^{\prime}}=\left(\pi_{0} v\right)^{\psi^{\prime}}=\pi_{0}^{\psi \tau} v^{\psi^{\prime}}=\pi_{0}^{\tau} v^{\psi}=\pi_{0} v .
$$

Consequently, $\pi_{0}^{\tau-1}=v^{1-\psi^{\prime}}$, whence $\pi_{0}^{\tau-1} V\left(F_{2} / F_{1}\right)=v^{1-\psi^{\prime}} V\left(F_{2} / F_{1}\right)$. We note that $u \rightleftharpoons N_{F_{2} / F_{1}}(v)=N_{F_{2} / F_{1}}\left(\pi_{0}^{\prime} \pi_{0}^{-1}\right)=N_{F_{2} / F_{1}}\left(\pi_{0}^{\prime}\right) N_{F_{2} / F_{1}}\left(\pi_{0}\right)^{-1} \in F$ because $N_{F_{2} / F_{1}}\left(\pi_{0}^{\prime}\right) \in$ $F_{1} \cap F_{0}^{\prime}=F$ and $N_{F_{2} / F_{1}}\left(\pi_{0}\right) \in F_{1} \cap F_{0}=F$. It follows that $u \in U_{R}^{F_{2}}$ and $\rho_{\psi}(u)=$ $\rho_{\psi^{\prime}}(u)=\pi^{\tau_{0}-1} V\left(F_{0} / F_{1}\right)$. Thus,

$$
\rho_{\psi}\left(U_{R}^{F_{2}}\right)=H^{-1}\left(G, U_{R_{2}}\right)\left(\simeq H^{-1}\left(G, U_{R_{0}}\right)\right) .
$$

The following lemma is important.

Lemma 1. $N_{F_{0} / F}\left(U_{R_{0}}\right) \leq U_{R}^{F_{2}}$ and $\operatorname{Ker} \rho_{\psi}=N_{F_{0} / F}\left(U_{R_{0}}\right)$.

This is a special case of [3, Proposition 6].

Corollary. $U_{R}^{F_{2}} / N_{F_{0} / F}\left(U_{R_{0}}\right) \simeq G$.

Lemma 2. $U_{R}^{F_{2}}=U_{R}$. 
Proof. If $\mathbb{F}_{1}^{\prime} \geq \mathbb{F}_{1}$ is also a finite unramified extension of $\mathbb{F}, F_{2}^{\prime} \rightleftharpoons F_{1}^{\prime} F_{0}=F_{1}^{\prime} \otimes_{F} F_{0}$, and $U_{R}^{F_{2}^{\prime}} \rightleftharpoons\left\{u \mid u \in U_{R}\right.$ is such that there exists $v \in U_{R_{2}^{\prime}}$ with $\left.N_{F_{2}^{\prime} / F_{1}^{\prime}}(v)=u\right\}$, then it can easily be verified that $U_{R}^{F_{2}^{\prime}} \geq U_{R}^{F_{2}}$. We have $U_{R}^{F_{2}} / N_{F_{0} / F}\left(U_{R_{0}}\right) \leq U_{R}^{F_{2}^{\prime}} / N_{F_{0} / F}\left(U_{R_{0}}\right)$. Moreover,

$$
U_{R}^{F_{2}^{\prime}} / N_{F_{0} / F}\left(U_{R_{0}}\right) \simeq G, \quad U_{R}^{F_{2}} / N_{F_{0} / F}\left(U_{R_{0}}\right) \simeq G .
$$

Since $G$ is finite, it follows that $U_{R}^{F_{2}}=U_{R}^{F_{2}^{\prime}}$.

Let $u \in U_{R}$. Then there exists a finite unramified extension $\mathbb{F}_{1}^{\prime} \geq \mathbb{F}$ such that $\mathbb{F}_{1}^{\prime} \geq \mathbb{F}_{1}$ and $u \in U_{R}^{F_{1}^{\prime}}$. Indeed, let $\overline{\mathbb{F}}_{1}$ be the maximal unramified extension of $\mathbb{F}$, and let $\bar{F}_{2} \rightleftharpoons$ $\bar{F}_{1} F_{0}$. Then, by condition 4 ), we have $N_{\bar{F}_{2} / \bar{F}_{1}}\left(\bar{F}_{2}^{\times}\right)=\bar{F}_{1}^{\times}$. In particular, there exists a finite subextension $\mathbb{F}_{1}^{\prime} \leq \overline{\mathbb{F}}_{1}$ such that $u \in N_{F_{2}^{\prime} / F_{1}^{\prime}}\left(F_{1}^{\prime \times}\right)$. We can choose $\mathbb{F}_{1}^{\prime}$ so that $\mathbb{F}_{1}^{\prime} \geq \mathbb{F}_{1}$. From the relations $u \in U_{R}^{F_{2}^{\prime}}=U_{R}^{F_{2}}$, it follows that $U_{R}^{F_{2}}=U_{R}$.

Corollary. $H^{0}\left(G, U_{R_{0}}\right)=U_{R} / N_{F_{0} / F}\left(U_{R_{0}}\right)=U_{R}^{F_{2}} / N_{F_{0} / F}\left(U_{R_{0}}\right) \simeq G$.

It remains to prove that $H^{0}\left(G, U_{R_{0}}\right) \simeq H^{0}\left(G, F_{0}^{\times}\right)$.

The exact sequence

$$
1 \longrightarrow U_{R} \longrightarrow F^{\times} \stackrel{v_{R}}{\longrightarrow} \Gamma_{R} \longrightarrow 1
$$

gives rise to the exact sequence

$$
\begin{array}{cc}
1 \longrightarrow U_{R} / N_{F_{0} / F}\left(F_{0}^{\times}\right) \cap U_{R} \longrightarrow F^{\times} / N_{F_{0} / F}\left(F_{0}^{\times}\right) \\
\longrightarrow \Gamma_{R / v_{R}}\left(N_{F_{0} / F} /\left(F_{0}^{\times}\right)\right) \longrightarrow c & 1 .
\end{array}
$$

Observe that $N_{F_{0} / F}\left(F_{0}^{\times}\right) \cap U_{R}=N_{F_{0} / F}\left(U_{R_{0}}\right)$. By Proposition 1.4, 1), we obtain $v_{R}\left(N_{F_{0} / F}\left(F_{0}^{\times}\right)\right)=f \Gamma_{R}$, where $f=\left[F_{R_{0}}: F_{R}\right]$. However, $f=1$ in the case in question, and we obtain $v_{R}\left(N_{F_{0} / F}\left(F_{0}^{\times}\right)\right)=\Gamma_{R}$. Then the exact sequence $(*)$ yields an isomorphism $H^{0}\left(G, U_{R_{0}}\right) \simeq H^{0}\left(G, F_{0}^{\times}\right)$.

Corollary. The class field theory axiom is fulfilled for the cyclic totally ramified extensions $\mathbb{F}_{0} \geq \mathbb{F}$ if $\mathbb{F}$ satisfies conditions 1 )-4) of the theorem.

\section{§4. Arbitrary CyClic extensions}

Proposition 4.1. Let $\mathbb{F}=\langle F, R\rangle$ be a valued field satisfying conditions 1$)-4)$ of the theorem. Let $\mathbb{F}_{0}=\left\langle F_{0}, R_{0}\right\rangle \geq \mathbb{F}$ be a cyclic extension, and let $G \rightleftharpoons G\left(F_{0} / F\right)=\langle\sigma\rangle$. Then $H^{-1}\left(G, F_{0}^{\times}\right)=1$ and $\left|H^{0}\left(G, F_{0}^{\times}\right)\right|=|G|$.

Proof. The fact that the group $H^{-1}\left(G, F_{0}^{\times}\right)$is trivial for a cyclic extension $F_{0} \geq F$ follows from Hilbert's Theorem 90.

Let $(F \leq) F_{1}$ be the maximal unramified subextension of $F_{0}$, and let $R_{1} \rightleftharpoons R_{0} \cap F_{0}$. Then $\mathbb{F}_{1} \geq \mathbb{F}$ is an unramified cyclic extension and $\mathbb{F}_{0} \geq \mathbb{F}_{1}$ is a totally ramified extension. Put $n_{0} \rightleftharpoons\left[F_{0}: F_{1}\right]$ and $n_{1} \rightleftharpoons\left[F_{1}: F\right]$. Then $n_{0} \cdot n_{1}=n \rightleftharpoons\left[F_{0}: F\right]=|G|$ and $G\left(F_{0} / F_{1}\right)=\left\langle\sigma^{n_{1}}\right\rangle$.

Now, we use the exact sequence $(*)$ (see $\S 3)$.

Proposition 1.4, 1) shows that $v_{R}\left(N_{F_{0} / F}\left(F_{0}^{\times}\right)\right)=f \Gamma_{R}$, where $f$ is the relative degree of $\mathbb{F}_{0} \geq \mathbb{F}$. Then $f=n_{1}, v_{R}\left(N_{F_{0} / F}\left(F_{0}^{\times}\right)\right)=n_{1} \Gamma_{R}$, and $\Gamma_{R} / n_{1} \Gamma_{R} \simeq C_{n_{1}}$.

We have $N_{F_{0} / F}\left(F_{0}^{\times} \cap U_{R}\right)=N_{F_{0} / F}\left(U_{R_{0}}\right)$. We prove that

$$
U_{R} / N_{F_{0} / F}\left(U_{R_{0}}\right) \simeq U_{R_{1}} / N_{F_{0} / F_{1}}\left(U_{R_{0}}\right) .
$$

By Proposition 2.1, the norm mapping $N_{F_{1} / F}$ from $U_{R_{1}}$ to $U_{R}$ is an epimorphism (because $\mathbb{F}_{1}$ is unramified over $\left.\mathbb{F}\right)$. Since $N_{F_{1} / F}\left(N_{F_{0} / F}\left(U_{R_{0}}\right)\right)=N_{F_{0} / F}\left(U_{R_{0}}\right)$, we see that $N_{F_{1} / F}$ 
induces an isomorphism between $U_{R_{1}} / N_{F_{0} / F_{1}}\left(U_{R_{0}}\right)$ and $U_{R} / N_{F_{0} / F}\left(U_{R_{0}}\right)$. Since $\mathbb{F}_{0} \geq F_{1}$ is a totally ramified cyclic extension, Proposition 3.2 implies the relations

$$
U_{R_{1}} / N_{F_{0} / F_{1}}\left(U_{R_{0}}\right)=H^{0}\left(G\left(F_{0} / F_{1}\right), U_{R_{0}}\right) \simeq G\left(F_{0} / F_{1}\right) .
$$

The exact sequence $(*)$ shows that

$$
\begin{gathered}
\left|H^{0}\left(G, F_{0}^{\times}\right)\right|=\left|F^{\times} / N_{F_{0} / F}\left(F_{0}^{\times}\right)\right|=\left|U_{R} / N_{F_{0} / F}\left(U_{R_{0}}\right)\right| \cdot\left|\Gamma_{R} / n_{1} \Gamma_{R}\right| \\
=\left|G\left(F_{0} / F_{1}\right)\right| \cdot n_{1}=n_{0} \cdot n_{1}=n=|G| .
\end{gathered}
$$

The proposition is proved.

The following theorem (stated in the Introduction) is a consequence of the above proposition:

For a valued field satisfying conditions 1)-4) of the theorem, local class field theory is valid.

Indeed, Proposition 4.1 shows that, in this case, the class field theory axiom is fulfilled for the $G(F)$-module $F_{\text {sep }}^{\times}$.

\section{§5. Sufficient conditions. An example}

Among conditions 1)-4) ensuring the validity of local class field theory, condition 4) is most difficult to verify. By [5], Chapter II, $\S 3$, Proposition 5], this condition is equivalent, in particular, to one of the following conditions:

$\left.4^{\prime}\right) \operatorname{Br}\left(F_{0}\right)=0$ for every finite separable extension $F_{0} \geq \bar{F}$;

$4^{\prime \prime}$ ) for all finite extensions $F_{0} \geq F_{1} \geq \bar{F}$ of $\bar{F}$, we have $N_{F_{0} / F_{1}}\left(F_{0}^{\times}\right)=F_{1}^{\times}$.

It is condition $4^{\prime \prime}$ ) that was used in $\S 3$.

Condition $4^{\prime}$ ) for $\bar{F}$ can be restated as follows: for every finite separable extension $\mathbb{F}_{0} \geq$ $\mathbb{F}$, each finite-dimensional skew-field $D$ over $F_{0}$ splits over a finite unramified extension $\mathbb{F}_{1} \geq \mathbb{F}_{0} ;$ moreover, it suffices to check this for the skew-fields such that $\left[D: F_{0}\right]=p^{2}$ for all prime $p$.

Proposition 5.1. Let a Henselian valued field $\mathbb{F}=\langle F, R\rangle$ be such that $F_{R}$ is perfect and $\Gamma_{R}$ is a z-group. Then every skew-field $D$ with center $F$ such that $[D: F]=p^{2}$ for a prime $p$ splits over an unramified extension $\mathbb{F}$ if one of the following conditions is fulfilled:

1) $F_{R}$ has characteristic 0 ;

2) $F$ is a maximal valued field (see [4, 6]);

3) $\Gamma_{R} \simeq \mathbb{Z}$, i.e., $\mathbb{F}$ is a discrete valuation field.

Proof. The Henselian valuation $v_{R}$ of $F$ can be extended uniquely up to a valuation $v_{D}$ of the skew-field $D$, and we have $[D: F]=\left[\bar{D}: F_{R}\right] \cdot\left[\Gamma_{D}: \Gamma_{R}\right] q^{k}$. Here, $\bar{D}$ is the residue algebra, $\Gamma_{D}$ is the value group of $v_{D}, q=1$ if the residue field $F_{R}$ has characteristic 0 , $q=\chi\left(F_{R}\right)$ if the characteristic $\chi\left(F_{R}\right)$ of $F_{R}$ is greater than 0 , and $k \geq 0$ is a positive integer [7].

To prove the proposition, it suffices to show that $\bar{D} \neq F_{R}$. Indeed, let $\alpha \in \bar{D} \backslash F_{R}$, and let $a \in R_{v_{D}}$ (= the valuation ring of $v_{D}$ ) be such that $\alpha=\bar{a}=a+\mathfrak{m}\left(R_{n_{D}}\right)$. Then $F_{0} \rightleftharpoons F(a) \leq D$ is a subfield of $D$ and a proper extension of $F$. From the results of 8 Chapter 13] it follows that the degree $\left[F_{0}: F\right]$ divides $p$. Then $\left[F_{0}: F\right]=p$ and $F_{0}$ is a maximal subfield of $D$, and therefore a splitting field of $D$. The degree $\left[F_{R}(\alpha): F_{R}\right]$ divides $\left[F_{R_{0}}: F_{R}\right]\left(R_{0} \rightleftharpoons R_{v_{D}} \cap F_{0}\right)$, and $\left[F_{R_{0}}: F_{R}\right]$ divides $\left[F_{0}: F\right]=p$. Since $F_{R}(\alpha) \neq F_{R}$, we have $F_{R}(\alpha)=F_{R_{0}},\left[F_{R_{0}}: F_{R}\right]=p=\left[F_{0}: F\right]$, and $F_{0}$ is an unramified extension of $F$.

For every $b \in D^{\times}$, we have $v_{D}(b)=v_{R_{1}}(b)$, where $R_{1} \rightleftharpoons R_{v_{D}} \cap F(b),[F(b): F]$ divides $p$, and $\left[\Gamma_{R_{1}}: \Gamma_{R}\right]$ divides $[F(b): F]$. Consequently, $p v_{R_{1}}(b) \in \Gamma_{R}$. It follows that the 
factor group $\Gamma_{D} / \Gamma_{R}$ has period $p$. Since $\Gamma_{R}$ is a $z$-group, we see that $\left[\Gamma_{D}: \Gamma_{R}\right]$ divides $p$ (i.e., $\left[\Gamma_{D}: \Gamma_{R}\right]=1$ or $p$ ).

If $q^{k}=1$, then we obtain the required relation $\bar{D} \neq F_{R}$. The identity $q^{k}=1$ is valid both in case 1) (because $q=1$ in this case) and in case 2) (by [6, Chapter 2, Theorem 11]).

Suppose condition 3 ) is fulfilled. We consider the completion $\widehat{\mathbb{F}}$ of $\mathbb{F}$ with respect to the topology defined by $v_{R}$; then $\hat{D} \rightleftharpoons D \otimes_{F} \widehat{\mathbb{F}}$ remains a skew-field (see [8]), and $\widehat{\mathbb{F}}$ satisfies condition 2) and $\bar{D}=\overline{\widehat{D}}$.

The proposition is proved.

Since conditions 1)-3) of the proposition are preserved under finite separable extensions, we arrive at the following statement.

Corollary. Under the conditions of Proposition 5.1, the maximal immediate extension $\bar{F}$ of $\mathbb{F}$ satisfies condition $\left.4^{\prime}\right)$.

The following proposition is yet another consequence of Proposition 5.1.

Proposition 5.2. Let $\mathbb{F}$ be a Henselian valued field such that $\Gamma_{R}$ is a z-group, $F_{R}$ is perfect, $G\left(F_{R}\right) \simeq \widehat{\mathbb{Z}}$, and one of conditions 1)-3) of Proposition 5.1 is fulfilled. Then local class field theory is valid for $\mathbb{F}$.

It suffices to observe that one of conditions 1), 2), and 3) of Proposition 5.1 and the fact that $\mathbb{F}$ is Henselian imply that $\mathbb{F}$ is algebraically complete.

In conclusion, we consider an example. Let $k$ be a perfect field such that $G(k) \simeq \widehat{\mathbb{Z}}$ (e.g., $k$ is a finite field or $k=\mathbb{C}\langle\langle t\rangle\rangle$ is the field of formal power series over the field $\mathbb{C}$ of complex numbers). Let $\Gamma \rightleftharpoons \sum_{p \in \mathcal{P}} \widehat{\mathbb{Z}}_{p}$ be the direct sum of the groups $\widehat{\mathbb{Z}}_{p} \simeq$ $\lim _{n \in \omega} Z / p^{n} Z$ (isomorphic to the additive group of $p$-adic integers; $\mathcal{P}$ is the set of all prime numbers). There is a natural embedding

$$
\Gamma \leq \widehat{\mathbb{Z}} \simeq \prod_{p \in \mathcal{P}} \widehat{\mathbb{Z}}_{p}
$$

We note that $\Gamma$ is a $z$-group, but it does not contain any generators of the group $\widehat{\mathbb{Z}}$. Since $\Gamma$ is a torsion-free Abelian group, the group $\Gamma$ can be linearly ordered. Viewing $\Gamma$ as a linearly ordered group, we can define a field $F \rightleftharpoons k\left\langle t^{\Gamma}\right\rangle$ (see [4, Chapter 1, §7], having a valuation $v: F^{\times} \rightarrow \Gamma$ such that $F_{R_{v}}=k$ and $\mathbb{F}=\left\langle F, R_{v}\right\rangle$ is a maximal valued field for which local class field theory is valid.

\section{REFERENCES}

[1] I. B. Fesenko and S. V. Vostokov, Local fields and their extensions. A constructive approach, Transl. Math. Monogr., vol. 121, Amer. Math. Soc., Providence, RI, 1993. MF,1218392 (94d:11095)

[2] J. Neukirch, Algebraic number theory, Grundehren Math. Wiss., vol. 322, Springer-Verlag, Berlin, 1999, Chapter IV. MR1697859 (2000m:11104)

[3] Yu. L. Ershov, Abstract class field theory (a finitary approach), Mat. Sb. 194 (2003), no. 2, 37-60; English transl., Sb. Math. 194 (2003), no. 1-2, 199-223. MR1992148 (2004f:11131)

[4] _ Multi-valued normed fields, "Nauchn. Kniga," Novosibirsk, 2000. (Russian)

[5] J.-P. Serre, Galois cohomology, Springer-Verlag, Berlin, 1997. MR,1867431|(2002i:12004)

[6] O. F. G. Schilling, The theory of valuations, Math. Surveys, vol. 4, Amer. Math. Soc., Providence, RI, 1950. MF $0043776(13: 315 b)$ 
[7] P. Draxl, Ostrowski's theorem for Henselian valued skew fields, J. Reine Angew. Math. 354 (1984), 213-218. MR0767581 (86g:12008)

[8] R. Pierce, Associative algebras, Grad. Texts in Math., vol. 88, Springer-Verlag, New York-Berlin, 1982. MR0674652 (84c:16001)

Sobolev Mathematical Institute, Akademik Koptyug Ave., Novosibirsk 630090, Russia

E-mail address: ershov@math.nsc.ru

Received 10/OCT/2002

Translated by B. M. BEKKER 